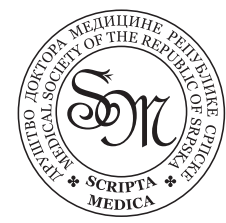

CASE REPORT

\title{
Fifty-Four-Year-Old Man with Inguinal and Testicular Masses
}

(Scr Med 2011;42:100-1)

\section{Yury Takhalov}

Department of Pathology

University of Illinois at Chicago and Stroger Hospital of Cook County

Chicago, IL 60612, USA

\section{Correspondence}

Yury Takhalov, MD

Department of Pathology, University of Illinois at Chicago

Chicago, IL 60612, USA

Submitted: July 13, 2011

Accepted: August 15, 2011
An extensive lipomatous growth was discovered in the inguinal region of a 54-year-old male during hernia repair surgery. The differential diagnoses included lipoma, angio-myolipoma, well differentiated liposarcoma and myxoid liposarcoma. After further work up, the diagnosis of well-differentiated liposarcoma seemed most credible, and total resection of the tumor was recommended.

A CT scan was done to establish the extent of the tumor. Figure 1. shows the CT scan of the patient with a mass on the left side in the inguinal region. There was no evidence of invasion.

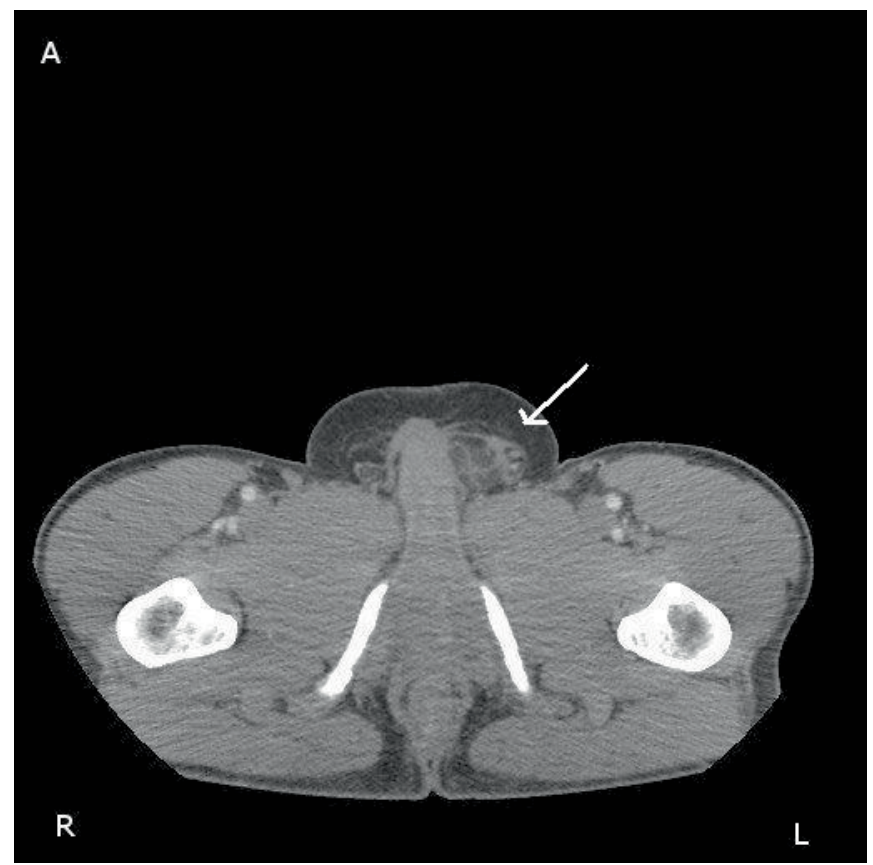

Figure 1. CT scan of the patient with a mass on the left side in the inguinal region.
Resection of the left inguinal canal and left orchiectomy was performed, and the gross specimen is shown in Figure 2. A well-circumscribed mass of $6.5 \mathrm{~cm}$ (A) within the tunica vaginalis displaced, but did not invade, the testicle (C) and extended into the spermatic cord. The mass traveled up the spermatic cord for $5.5 \mathrm{~cm}$, connecting to a second mass of $4.5 \mathrm{~cm}$ (B) within the inguinal region.

Tissue sections viewed under low power microscopy showed dense fibrotic zones alternating with mature adipocytes of varying size (Figure 3). Examination under higher power showed atypical pleomorphic stromal cells within the dense fibrotic zones (Figure 4). Our final diagnosis was well-differentiated liposarcoma, sclerosing type.

Well-differentiated liposarcomas of any type have no potential for metastasis unless they undergo dedifferentia-

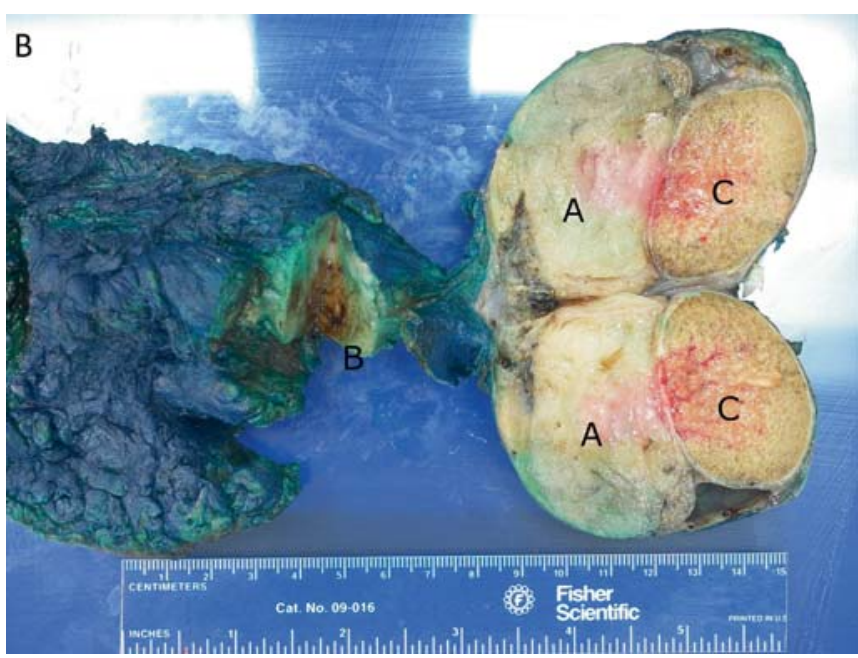

Figure 2. Gross specimen, after resection of the left inguinal canal and left orchiectomy, is presented. 


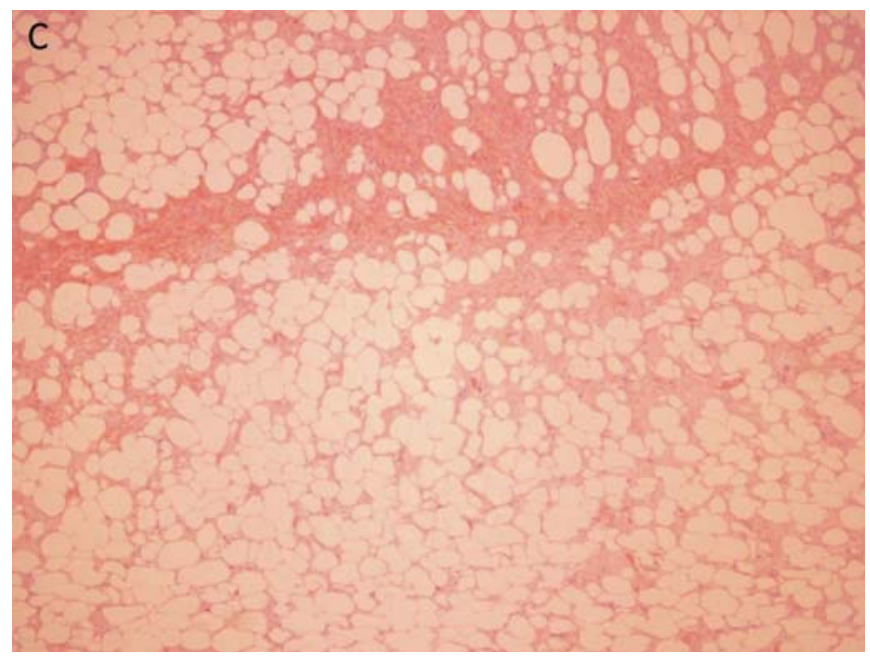

Figure 3. Tissue sections viewed under low power microscopy showed dense fibrotic zones alternating with mature adipocytes of varying size.the inguinal region.

tion. ${ }^{1}$ They are generally found in adults between the ages of $40-60$. The most common location for this tumor is within the lower limbs, retroperitoneum, paratesticular and mediastinal regions. They can be circumscribed or infiltrative; on gross examination appear as bulging, large, yellow white tumors, coarsely lobulated with pale, firm areas. Microscopically, they exhibit mature, variably sized adiposities and fibromyxoid stroma that contains spindle cells with large, dark staining nuclei along with marked

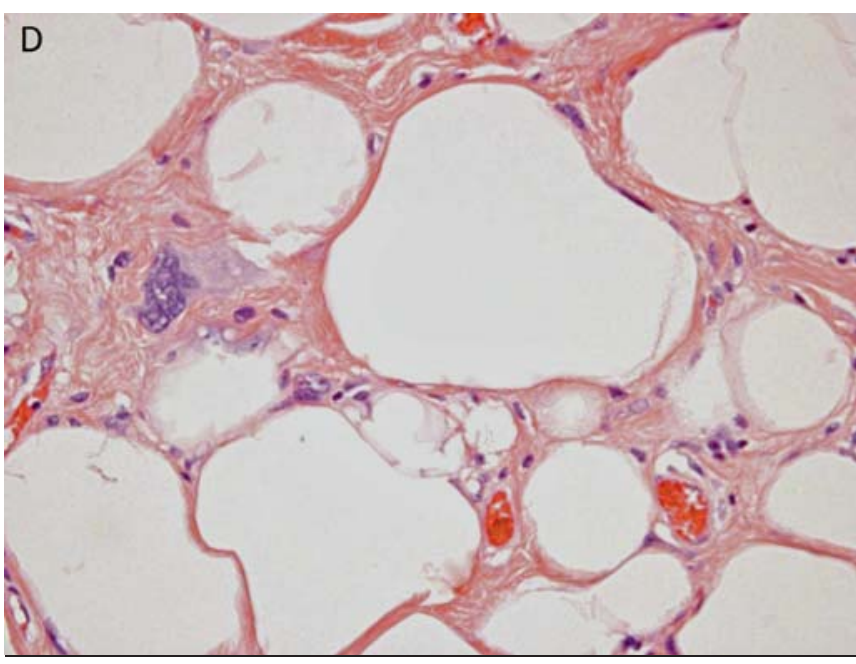

Figure 4. Examination under higher power showed atypical pleomorphic stromal cells within the dense fibrotic zones.

nuclear enlargement and pleomorphism. The cellularity is usually low, and mitotic figures are rare. Lipoblasts may be obvious, but these cells are not essential to confirm the diagnosis of liposarcoma.

\section{Reference}

1. Laurino L, Furlanetto A, Orvieto E, Dei Tos AP. Well-differentiated liposarcoma (atypical lipomatous tumors). Semin Diagn Pathol 2001;18:258-62. 